\title{
The evidence indicates that the hamstrings do not behave isometrically during the swing phase of the sprint cycle: a narrative review
}

\author{
Judd T. Kalkhoven, ${ }^{1}$ Deborah L. Sides, ${ }^{2}$ Blake D. McLean, ${ }^{1}$ \& Mark L. Watsford ${ }^{1}$ \\ ${ }^{1}$ Human Performance Research Centre, Faculty of Health, University of Technology Sydney, \\ Australia \\ ${ }^{2}$ English Institute of Sport, Manchester Institute of Health and Performance, United \\ Kingdom
}

June 8, 2020 (Version 2)

\begin{abstract}
In opposition to the majority of research, it has recently been suggested that the hamstrings behave isometrically during the swing phase of sprinting. Despite little evidence, this theory postulates that hamstring muscle slack enables passive hamstring lengthening during the initial- and mid-swing phases of sprinting. It has also been theorised that the hamstrings then contract isometrically in the late-swing phase whilst the hamstring tendons stretch and store elastic energy. There are a number of deficiencies with this theory. Evidence from electromyogram studies demonstrate that the hamstrings are active during the entirety of the sprint cycle, indicating that hamstring muscle slack does not exist whilst sprinting and that hamstring lengthening occurs eccentrically. Additionally, there are substantial mechanical benefits to eccentric muscle behaviour that do not present under isometric conditions, such as force enhancement, force augmentation per unit of energy cost, and higher contraction velocities through sarcomere recoil. All of which are expected to contribute to hamstring muscle functioning whilst sprinting. Kinematically, joint velocity and acceleration profiles further refute the idea that the hamstring is functioning isometrically when sprinting. Kinetically, hamstring forces exceed isometric capacity during the late-swing phase of sprinting, dictating that eccentric contributions are required in this phase. Collectively, the evidence indicates that passive and isometric behaviour of the hamstrings in the swing phase of sprinting is unlikely, with the hamstrings acting eccentrically instead. Based on the current evidence, suggestions that isometric exercises should be prescribed on the basis that they mimic hamstring muscle function whilst sprinting, are unreasonable and should be dismissed.
\end{abstract}

Keywords: Muscle function; Eccentric; Muscle slack; Force enhancement; Sarcomere recoil

DOI: http://dx.doi.org/10.31236/osf.io/8ujt2

Citation: Kalkhoven, J. T., Sides, D. L., McLean, B, D., \& Watsford, M. L., (2020). The evidence indicates that the hamstrings do not behave isometrically during the swing phase of the sprint cycle: a narrative review. SportRxiv. Doi:10.31236/osf.io/8ujt2

Corresponding author: Judd Kalkhoven (Judd.kalkhoven@uts.edu.au) This work is a preprint and has yet to be peer-reviewed.

All authors have read and approved this version of the manuscript for pre-print. Twitter handles: @KalkhovenJudd / @DebsSides / @Blake_Mc_ 


\section{TABLE OF CONTENTS}

1. Introduction

2. "Muscle slack"

3. Muscle mechanics: eccentric muscle behaviour and the role of intramuscular passive filaments

4. Kinematics and hamstring kinetics whilst sprinting

5. Contraction velocity and sarcomere recoil

6. Musculoskeletal modelling and animal studies

7. Conclusion

\subsection{INTRODUCTION}

It has recently been theorised that the hamstrings behave isometrically during the swing phase (Figure 1) of sprinting [1-3]. Specifically, the isometric hamstring phenomenon described by Van Hooren \& Bosch [1] suggests that following passive lengthening of the hamstring musculature in the initial- and mid-swing phases of sprinting, the hamstring behaves isometrically in the late-swing phase immediately prior to ground contact. These suggestions have arisen despite an absence of supporting evidence, along with the majority of published research suggesting that the hamstrings behave eccentrically in the swing phase [416]. The isometric theory is primarily purported by one research group, with three recent articles [1-3] proposing muscle slack and tendon stretch as primary contributors to the occurrence of the described muscle behaviour.

Considering that the late-swing and early-stance phases are widely regarded as the primary stages of sprinting during which hamstring injuries occur [10, 12], theories surrounding function and contraction mode have the capacity to greatly influence understandings of hamstring injury mechanisms. These conceptual frameworks impact hamstring training and injury mitigation protocols. Indeed, the belief that the hamstrings behave isometrically whilst sprinting has resulted in a variety of isometric hamstring exercises proposed as being most efficacious for injury prevention [2, 17]. Most notably, in response to these suggestions isometric exercises have gained considerable traction within the strength and conditioning community for both hamstring injury risk mitigation $[2,18,19]$ and athlete strength development [3, 17-19].

The purpose of this article is to highlight the theoretical flaws in the recent suggestions that the hamstrings lengthen passively prior to behaving isometrically during the late-swing phase of the sprint cycle [1-3]. Indeed, the evidence suggests an absence of hamstring muscle slack (and therefore passive lengthening) throughout the entirety of the sprint cycle. With consideration to mechanical muscle function, sarcomere behaviour, energy cost, contraction velocity as well as kinematics and hamstring kinetics while sprinting, the collective evidence indicates eccentric muscle action during the swing phase of sprinting. This paper examines each of these aspects to build upon the conceptual understanding of hamstring function during sprinting. These concepts have implications for understanding injury mechanisms and improving exercise prescription for injury reduction and rehabilitation.

\section{0 "MUSCLE SLACK"}

The term "slack" is most commonly used to describe a material that is not taut. However, "muscle slack", the removal of which is purported to include alignment of the musculotendinous unit (MTU), the uptake of slack in the aligned contractile element (CE) and series elastic component (SEC), changes in 3-dimensional muscle shape, and SEC compliance $[1,20]$, has been

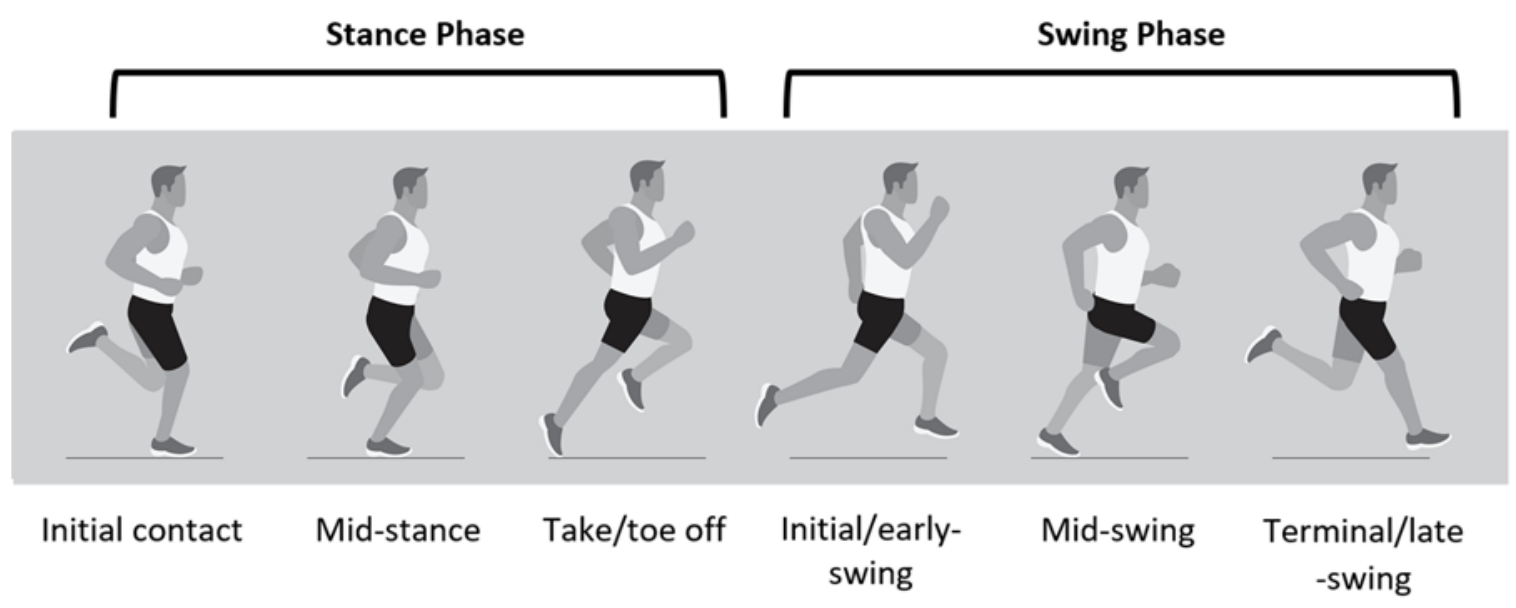

Figure 1: The phases of the sprint cycle 
used by Van Hooren \& Bosch [20] to describe the delay between the start of CE activation and SEC recoil. This description requires deeper critical evaluation as such a definition would implicate intramuscular passive filament stretch, a primary contributor to the increased tensions exhibited in eccentric contractions, with a slack removal process. Accordingly, slack should preferably refer to the period preceding both recoil and the increase in potential energy within elastic elements, whereby 'slack' must first be removed for the SEC to stretch elastically [20, 21]. Despite these descriptive concerns, muscle slack has been cited as a primary mechanism that enables the passive lengthening of fascicles during the initial- and mid-swing phases of the sprint cycle [1-3].

It is further suggested that following passive lengthening of the musculature during the mid-swing phase, permitted by the existence of muscle slack, the hamstring contracts isometrically in the late-swing phase of sprinting [1]. Indeed, one of the key criticisms made of research studies that claim a high reliance on eccentric hamstring function whilst sprinting is the constant presence of muscle activation [1]. Van Hooren $\&$ Bosch contend that this negates the presence of slack within these models, leading to the misconception that the hamstrings are lengthening eccentrically during the swing phase [1]. However, these criticisms are unfounded as electromyography (EMG) data directly measuring the electrical activity of the hamstrings whilst sprinting contradict these suggestions [13, 22]. Indeed, these studies revealed that the hamstrings remain active during the entirety of the gait cycle in sprinting [13, 22]. Specifically, Jönhagen et al. [22] demonstrated that the minimum activation levels whilst sprinting were $24 \%$ and $27 \%$ of maximal EMG for the lateral and medial hamstrings, respectively. These findings are reflective of the typical agonist-antagonist relationship seen in this muscle group during high intensity activities such as maximal leg extension [23] and kicking [24], with the active participation of the antagonist muscle playing an important role in the control of posture and movement [25], as well as utilisation of the stretch-shorten cycle [25].

Considering that minimal tensions exist in either the SEC or CE until slack is removed across the length of the MTU, and that even low levels of muscle activation (below 5\% of maximum) increase MTU tensions [26], it is apparent that the hamstrings are in a constant state of tension during the sprint cycle. As such, hamstring muscle slack is indicatively non-existent whilst sprinting, as muscle activation levels would need to completely dissipate to alleviate these forces and allow for the reintroduction of slack into the MTU. Furthermore, the electrical activity of the hamstrings rapidly increases during mid-swing, with the greatest activation occurring during the late-swing and earlystance phases [13, 22]. Considering this activation pattern, it is inconceivable that hamstring "slack" could be present during the mid-swing phase of the sprint cycle as this would mean that the hamstrings experience minimal tensions whilst being relatively highly activated, an unrecorded phenomenon. These findings indicate that muscle slack is negligible within the hamstrings during the entirety of the sprint cycle and hamstring lengthening is conducted under active conditions.

Of additional interest, if muscle slack was indeed present and the hamstrings passively lengthened during mid-swing, it would not be possible for elastic energy to be stored in either the tendons or the musculature prior to the removal of slack from the MTU [27-30]. Until slack is removed across the length of the musculature, the tendon does not have an opposing force that enables it to stretch and gain potential energy [30]. Therefore, if muscle slack was present, minimal elastic energy would be stored across the entirely of the MTU during the mid-swing phase of sprinting, despite an expectedly large change in hamstring MTU length $[4,5]$. Finally, if muscle slack was indeed present during the mid-swing phase of the sprint cycle, the risk of hamstring muscle strain injury in this phase would be alleviated as minimal tensions would be experienced across the MTU until the slack is removed $[28,29]$.

\subsection{MUSCLE MECHANICS: ECCENTRIC MUSCLE BEHAVIOUR AND THE ROLE OF INTRAMUSCULAR PASSIVE FILAMENTS}

Over the past century a wealth of knowledge has been gathered within the field of muscle mechanics and functioning. As such, it is imperative that this knowledge is utilised when forming theoretical frameworks for muscle behaviour within applied settings. One such relevant concept to hamstring functioning whilst sprinting is that muscles which are forced to lengthen whilst active can sustain very high loads [31]. This notion is well-established, with Katz [31] demonstrating over 80 years ago that a muscle which is actively lengthening can produce forces up to 1.8 times greater than those observed during isometric or concentric contraction (figure 2).

Further, the force-velocity relationship of muscle provides interesting insights. For a muscle that is actively lengthening at only $1 \%$ of its maximum unloaded shortening velocity, an increase in tension of $50 \%$ of the maximum isometric tension can be achieved [31]. The discontinuity of the force-velocity relationship around zero velocity is such that tension rises approximately 10 times faster for lengthening than for an equivalent velocity of shortening [32]. These differences in behaviour can be attributed to contributions from passive filaments within and across sarcomeres, such as the winding filament titin, engaging during muscle activation and increasing tensions during active muscle stretch [33-36]. 


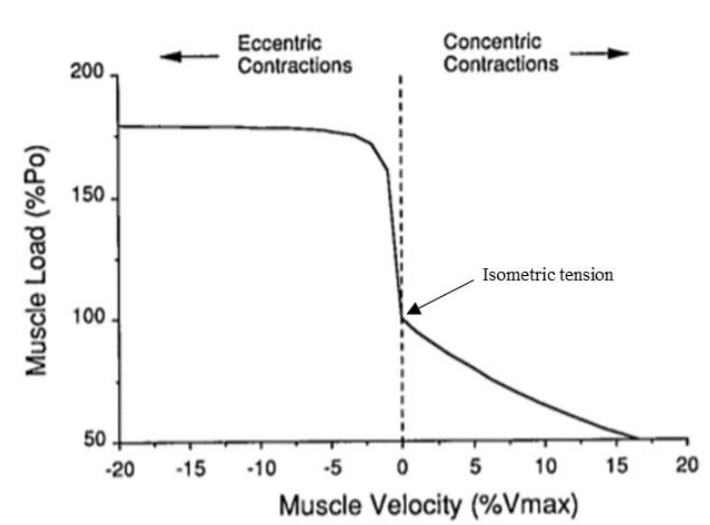

Figure 2: The classic force-velocity relationship. Note that eccentric force production is considerably greater than isometric and concentric force production. Po, isometric tension; \%Vmax, percent of maximal shortening velocity. Adapted from Fridèn \& Lieber [32] with permission.

In addition to the notably large tensions produced through passive filament stretch, this added tension is not associated with metabolic energy consumption [33]. As such, by acting eccentrically, the hamstring musculature can achieve the same, or greater, forces than isometric contractions while activating fewer sarcomeres, a known occurrence of muscle functioning [37-40]. In fact, the low metabolic cost of eccentric muscle contractions has been demonstrated [37, 41] with eccentric contractions exhibiting superior force outputs for a given energy cost [37-39, 41-43]. Accordingly, by acting isometrically when sprinting, the hamstring would be required to actively resist eccentric muscle lengthening to accommodate stretch of the tendons at an increased energy cost for a given force output [37-40]. This notion holds even greater relevance when considering sarcomere recoil $[33,36$, 44-46], another muscular phenomenon which will be discussed later in relation to contraction velocity.

Although the above-mentioned passive filament contributions were not included in the original model of skeletal muscle by Hill [47], it was acknowledged that muscle elasticity (series and non-series) might also reside in components other than the tendons, such as the muscle fibers themselves [48]. As research into muscle functioning has advanced, the existence of these intramuscular passive filaments and their contributions to force enhancement during eccentric muscle lengthening [33-36, 49] and the stretch shorten cycle $[46,49,50]$, has become largely accepted. Although it remains uncertain as to where exactly specific passive filaments fit within the context of the Hill model [49], some of these passive filaments, such as titin, primarily engage with greater levels of $\mathrm{CE}$ activation [33, 34, 36]. Accordingly, it is evident that muscle elasticity and the $\mathrm{CE}$ do not function exclusively of one another but are rather interdependent. Notably, some of these passive filaments have even been touted as potentially forming part of the SEC [51, 52]. As such, investigating MTU behaviour using a simplified Hill model, whereby
MTU elasticity is considered as merely the tendon and aponeurosis, and the contributions and engagement of intramuscular passive filaments upon activation are not considered within the slack removal process, or are ignored entirely, is inadequate. This additional knowledge is particularly relevant within the context of sprinting considering that the muscles are active during the entirety of the sprint cycle [13, 22], indicating that both active and passive filaments are engaged and tensions are being produced, negating the presence of muscle slack. Accordingly, the expectedly large hamstring MTU lengthening that occurs whilst sprinting [4, 5], must be considered under these specific active conditions.

When assessing muscle behaviour at the sarcomeric level, it is worth noting that while sarcomere lengths typically vary by approximately $1 \%$ across a single muscle fiber under passive conditions [53], these variations in length increase during muscle activation $[54,55]$. Interestingly, under fixed-end tetani, whereby a muscle is forced into isometric contraction through clamping, some sarcomeres alter in length independently [54, 55]. Specifically, under tetanic conditions, the actively weaker sarcomeres of isolated muscle fibers actually lengthen (a phenomenon referred to as creep) while the actively stronger sarcomeres of the fibers shorten [54]. This phenomenon is the result of discrepancies in the active force production capabilities of sarcomeres [32, 53, 54] and occurs because the actively weaker sarcomeres are required to lengthen (creep) and behave slightly eccentrically to take up passive tension. This is necessary to balance the forces across sarcomeres and maintain an isometric contraction $[32,53,54,56]$. The non-uniformity of sarcomeres has been established for decades [53-55, 57, 58] with non-uniform strain even being shown at more macroscopic levels, including across single muscle fibers [59], fascicles [60] and different regions of a given muscle [61]. It is therefore highly likely that groups of fascicles, or even portions of a given fascicle, behave variably across the hamstring musculature during the swing phase of the sprint cycle, especially when considering the varying architectural profiles that exist within certain hamstring muscles, such as biceps femoris long head [62, 63].

Independent of hamstring muscle function while sprinting, it is worth noting that although the term 'isometric' is commonly used to describe an active muscle state, whereby the muscle is not changing in length at a macroscopic level, it holds substantial limitations when describing detailed muscle behaviour. This is particularly the case regarding the internal structures of the musculature, such as sarcomeres, as a plethora of sarcomere force-length and force-velocity combinations exist within the muscle [32], as depicted in Figure 3. Indeed, considering that isometric contractions can present at different muscle lengths [64], even at a macroscopic level a variety of forcelength relationships can exist under isometric 


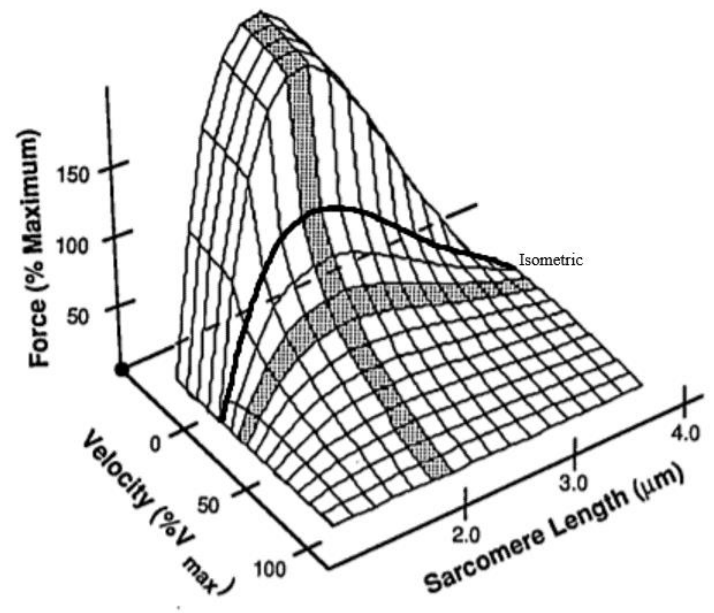

Figure 3: 3D graph depicting a generalized relationship between sarcomere length, force, and velocity within a single sarcomere. An entire muscle presents with a similar relationship profile. Note the multitude of tensions (black line) that can be achieved within a single sarcomere during isometric behavior. Adapted from Fridèn \& Lieber [32] with permission.

conditions [32, 64]. Accordingly, the term 'isometric' only appropriately serves to describe a muscle as being at a fixed length at the macroscopic level, which is an important consideration when attempting to describe or replicate muscle functioning in a detailed, relevant manner.

\subsection{KINEMATICS AND HAMSTRING KINETICS WHILST SPRINTING}

The swing phase is inherently important to sprint performance as the mechanically efficient swing of the limb sets up the other phases of the running stride for higher levels of mechanical efficiency [14]. An understanding of the joint and limb kinematic profiles for the entirety of the stride can provide valuable insights into muscle behaviour while sprinting. Specifically, hip and knee joint velocity-time curves (Figure 4), as presented by Sides and colleagues [14] for a sample of 20 international and national level sprinters, provides data to support the notion that the hamstrings do not behave isometrically whilst sprinting. Considering the biarticular nature of the hamstrings, most relevant to this paper is that neither the hip or knee joint angular velocity's cross the zero mark on the x-axis of the velocity-time graph at the same time. This demonstrates that there is movement occurring at either the hip or knee joint when sprinting at any given point in time, an unlikely phenomenon to occur under isometric hamstring conditions. Although it has been suggested that changes in MTU length cannot necessarily be attributed to muscle lengthening, and may be attributed to tendon stretch and recoil [1], as mentioned previously, intramuscular passive filaments are touted as forming part of the SEC [51, 52], and the stretch of intramuscular passive filaments during MTU lengthening is common place, augmenting a range of benefits for muscle functioning. As such, their exclusion is unlikely. Accentuating this unlikelihood is that if the hamstring musculature were to behave isometrically, the hamstring muscle would also be required to actively resist lengthening at high energy cost, negatively impacting both energy consumption and movement efficiency [65]. The improbability of isometric hamstring functioning is further amplified when considering that hip extension velocity begins to increase rapidly in mid- and lateswing, whilst the knee joint is predominantly extending [14], as shown in Figure 4. To generate high intensity

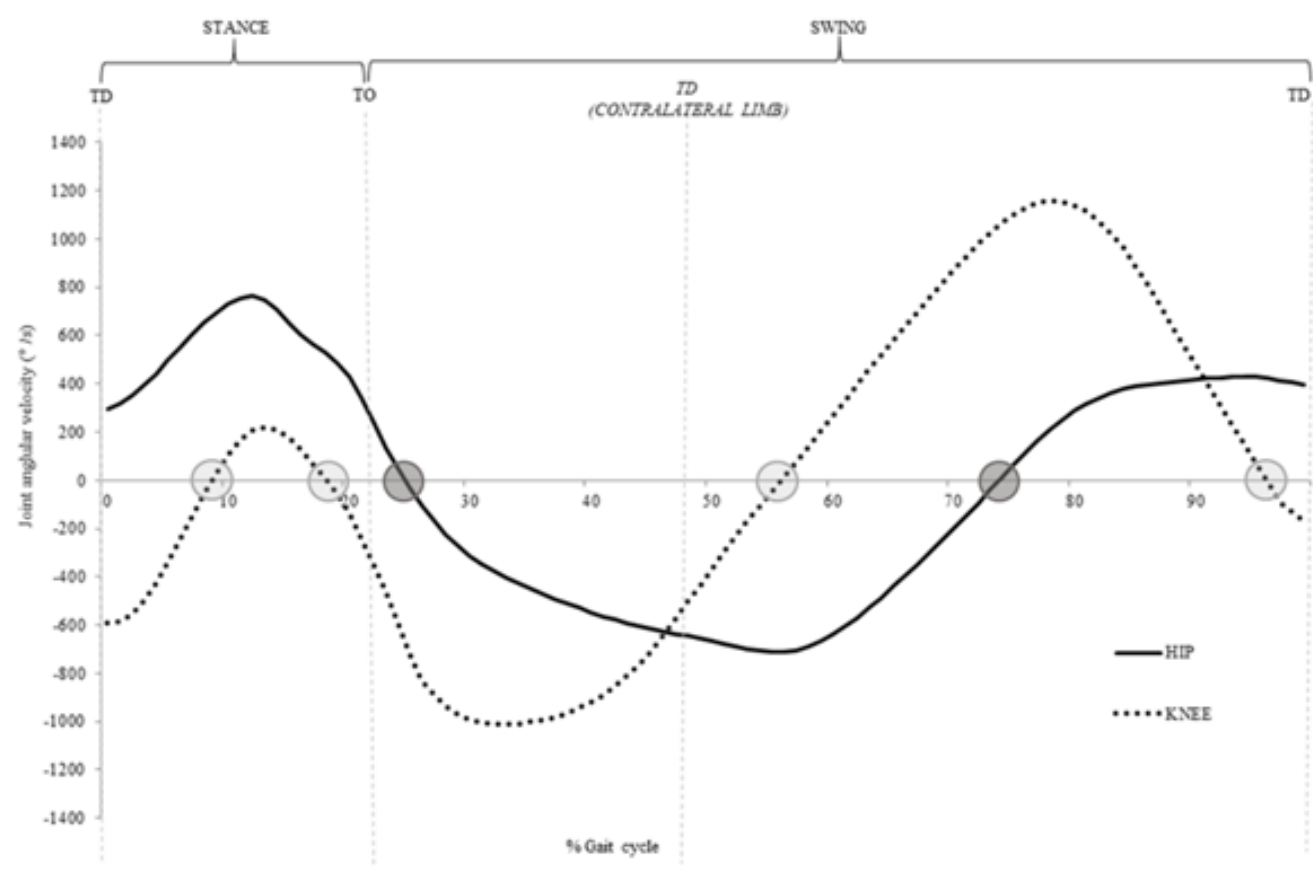

Figure 4: Joint angular velocity profile of a full gait cycle of the knee and ankle joints for elite sprinters. TD, Toe Down; MS, Midstance; TO, Toe Off. Note that hip and knee joint velocities never reach zero at the same time nor does the gradient of the curve alter at the hip or knee during transition from concentric to eccentric. Adapted from Sides [14] with permission. 
movement at both the hip and knee joints simultaneously whilst the hamstring behaves either passively or isometrically appears implausible. Although the gluteal muscles contribute to hip joint extension velocities $[13,22,66]$, it is unlikely that the gluteal muscles generate all of the movement across the hip at these high intensities [67], as this would require a unique dissociation of the hamstring and gluteal muscles while sprinting. This is atypical of the normal gluteal-hamstring relationship that has previously been documented [67].

Technically, any transition from eccentric to concentric contraction and vice versa, may be described as an "isometric" event. However, when ignoring the biarticular nature of the hamstrings, and the premise that movement is occurring at either the hip or knee joints at any given point in time, this transition phase instant is so small at either the knee or hip joint whilst sprinting that it is by all means insignificant. The triviality of the isometric instant is indicated by the fact that in elite sprinters, during the transition of the knee joint from extension to flexion immediately prior to toe down, angular acceleration at the knee joint is $~ 69$ deg.s $\mathrm{s}^{-2}$ [14]. Accordingly, this isometric instant is by all means imperceptible as the data frequency used (300 frames per second) by Sides et al. [14] to capture angular velocity could not identify a zero velocity point. Considering that the gradient of a velocity-time graph represents acceleration, the high accelerations which bring about the negligible 'isometric' instant are represented in Figure 4 by the gradient of the velocitytime curve at zero velocity. Note that this gradient remains virtually unchanged at either the knee or hip joints whilst the joint angle velocities cross the zero point on the $\mathrm{x}$-axis, indicating a smooth transition between contraction modalities with a negligible isometric instant. Regardless, the above-mentioned transition from eccentric to concentric muscle behaviour is a different phenomenon to the isometric contraction described in the isometric hamstring literature, as these authors theorised an absence of eccentric hamstring lengthening in favour of passive hamstring lengthening in mid-swing prior to a isometric contraction in late-swing [1].

Interestingly, when assessing the joint-angle profile of elite sprinters in the stance phase of sprinting, this phase includes slight knee flexion prior to a second extension during toe off $[4,14,68]$. This may have implications for elastic energy storage and the subsequent usage of this elastic energy within the proceeding early-swing phase. Regardless, during the stance phase, as the knee joint transitions from extension to flexion, then back to extension, the velocity-time gradient does not change as the velocity curve crosses zero. This indicates that even during stance, when minimal relative knee angle changes occur [14], the hamstrings do not behave isometrically.
At maximal running speeds when the body is vertical, the intense rearward movements of the lower limbs are necessary during both the late-swing and stance phases, with the hamstrings producing very high forces in both these phases [69]. A high extension velocity of the hip and knee in late-swing aids in achieving the optimum lower limb position at touchdown and increases the chance of negative foot speed relative to the centre of mass, which in turn, reduces the braking forces experienced [14]. This transition between swing and stance is of crucial importance for the hamstrings as they counteract both external hip flexion and knee extension moments, as well as withstand forces as high as 8 times body weight [15], which is beyond the maximal isometric force production capabilities of the hamstrings [15].

From a kinetic perspective, an isometric contraction is unlikely when considering that hamstring forces during the late-swing and early-stance phases have been reported to be as high as 8 times body weight [15]. It is not possible for these forces to be produced under isometric conditions and without eccentric contributions, especially when considering the maximal isometric forces commonly produced by the hamstrings $[15,70]$. Indeed, in elite male sprinters, the maximal muscle torque at the knee joint has been shown to be $\sim 1.4$ times the maximal isometric knee flexion torque that these athletes could actively produce [15]. The plausibility of producing the required forces necessary to behave isometrically in the swing phase is even less likely when considering the knee is largely extended during late-swing [14], placing the hamstrings in a less than optimal position on the force-length curve [71]. As such, this evidence supports previous propositions that the hamstrings behave eccentrically $[4,5]$, taking up both active and passive tensions (through active muscle stretch) to cope with the large forces experienced in the late-swing phase.

\subsection{CONTRACTION VELOCITY AND SARCOMERE RECOIL}

In addition to enhanced force production and energy efficiency, eccentric muscle stretch can augment concentric contraction velocities via sarcomere recoil $[33,37-39,41-43,46,50,72]$. It is widely accepted that stretch and recoil of the intramuscular passive filaments contributes substantially to the stretch shorten cycle $[49,50]$, and although utilisation of this mechanism has not been explored directly in humans whilst sprinting, evidence exists regarding the utilisation of sarcomere recoil for the enhancement of muscle contraction velocities [33, 34, 46, 72, 73]. Indeed, the winding filament titin [33, 46, 72] is responsible for the majority of the passive tension generated in musculature during active muscle stretch $[33,45]$, and is often described as a "molecular spring" $[33,46,74]$. This spring behaviour presents when the 
winding filament is extended during eccentric stretch prior to recoiling elastically, augmenting shortening behaviour of the musculature $[46,72,73]$. It has been demonstrated that in the early stages of myofibril shortening, the passive recoil speed of eccentrically lengthened myofibrils greatly exceeds the maximum active shortening velocities of previously unloaded sarcomeres [46]. Further, when titin functioning is impaired, negative work is reduced [50], indicating reduced energy storage by muscles during stretch and energy dissipation during shortening [50]. Given the cumulative benefits of sarcomere recoil in clinical studies, including enhanced contraction velocities at relatively low energy cost [33, 34, 46, 72, 73], utilisation of this mechanism likely explains the high velocities that can be achieved during powerful athletic movements, such as sprinting, and further exploration regarding the utilisation of this mechanism in sprinting is warranted.

Considering the energy efficiency of passive elastic contributions and heightened contraction velocities achievable through active muscle stretch and recoil, it is unlikely that the hamstrings would favour isometric behaviour during the sprint cycle, as this would negate the use of these mechanisms that have shown to enhance muscle function. If the hamstring muscles were to behave passively, then isometrically during the swing phase of sprinting, this would have considerable negative implications for hamstring contraction velocity capabilities during the remainder of the cycle. Considering the minimal additional knee extension after late-swing and toe down, if the hamstrings did not behave eccentrically during the swing phase they would be required to generate the hamstring contraction velocity exhibited during knee flexion in the proceeding swing phase entirely through active energy contributions. This would negatively impact hamstring contraction velocities due to the absence of fundamental intramuscular contributions to the stretch shorten cycle, namely sarcomere recoil. Further, the absence of active muscle stretch and sarcomere recoil within the sprint cycle would inevitably result in a heightened energy cost for a given contraction velocity due to a lack of elastic energy contribution within the muscle [37, 46].

\subsection{MUSCULOSKELETAL MODELLING AND ANIMAL STUDIES}

In support of the theory that the hamstrings behave isometrically [1], two modelling studies were cited that investigated hamstring function during sprinting $[4,5]$. Interestingly, both of these modelling studies actually suggested that the hamstrings behaved eccentrically during the mid- and late-swing phase of sprinting [4, 5]. To counter this, Van Hooren \& Bosch [1] focused on the limitations of these studies, citing tendon stiffness inputs that were too high as being a major contributing factor to the eccentric outcomes. However, in a clear shortcoming of the isometric argument, no evidence was provided that tendon stiffness was too high, nor was there an objectively supported rationale as to why the hamstrings should alternatively behave isometrically in these models.

An alternative rationale provided by Van Hooren \& Bosch [1] was centred on evidence from animal studies, suggesting that the biceps femoris behaves isometrically during gait in dogs [75] and goats [76]. Although these studies suggest isometric behaviour at the fascicular level in some phases of the gait cycle, extrapolating quadrupedal animal function to human biceps femoris action is inappropriate for a number of reasons. The quadrupedal nature of movement (i.e. completely different gait), the lower body weights of the mammals used in these studies, along with the methodological limitations of sonomicrometry used to assess strain across an entire muscle in these studies each contribute to substantially limit the application of such concepts in humans. Each of these limitations were indeed acknowledged by the original researchers $[75,76]$. Most notably, however, is the considerably different functions of the hamstring musculature in dogs and other quadrupedal mammals compared to humans [77, 78]. Markedly, the biceps femoris muscle of dogs is primarily designed for hip extension, exhibiting an insertion at the tibial tuberosity, and a markedly low moment arm at the stifle (knee) joint of dogs $[77,78]$. This is in contrast to the biceps femoris and semimembranosus muscles in humans, which have a relatively large moment arm across the knee joint [67]. This indicates the heightened role of the hamstrings in knee joint motion for humans relative to quadrupeds. The above-mentioned factors compound to stipulate that the extrapolation of these findings to human muscle behaviour is unbefitting.

\subsection{CONCLUSION}

It has been suggested that following passive lengthening in the mid-swing phase, the hamstrings behave isometrically during the late-swing phase of sprinting [1-3]. The authors of this theory cite muscle slack and the storage of elastic energy within the tendons as the primary contributors to this phenomenon [1]. However, the principal concepts underpinning this theory are unfounded, and empirical evidence strongly supports theories of eccentric muscle behaviour during this phase, as is typical of the agonistantagonist relationship commonly seen in the thigh 
musculature during high intensity leg extension activities. Indeed, evidence via EMG studies demonstrate that the hamstrings remain active across the entirety of the sprint cycle, meaning muscle slack does not exist, nor does passive muscle lengthening occur, at any time point during the sprint gait cycle.

To behave isometrically in the late-swing phase of the sprint cycle, the hamstrings would have to: 1 ) resist eccentric muscle lengthening to accommodate stretch of the tendons at an increased energy cost for a given force output, 2) produce active shortening with minimal intramuscular passive filament contributions at a high energy cost within the proceeding swing phase, and 3) generate movement at the hip and knee joints solely in the tendons of the hamstring whilst the hip joint experiences high angular velocities. Further, if all of these conditions were met, this would impair achievable contraction velocities and increase energy expenditure throughout the remainder of the sprint cycle. In addition to these kinematic considerations, the forces to which the hamstrings are exposed during the late-swing and early-stance phases of sprinting are extremely high and exceed the capacity of the muscle to function isometrically, especially at the muscle lengths experienced during these phases. Although it remains theoretical, the evidence overwhelmingly indicates that the hamstrings eccentrically lengthen during the swing phase of sprinting, as suggested by the vast majority of researchers working in this area [4-16]. Although isometric hamstring exercises may still hold value for conditioning and injury prevention purposes, based on the current evidence suggestions that isometric exercises should be prescribed on the basis that they mimic hamstring muscle function whilst sprinting, are unreasonable and should be dismissed.

\section{REFERENCES}

1. Van Hooren B, Bosch F. Is there really an eccentric action of the hamstrings during the swing phase of high-speed running? part I: A critical review of the literature. J Sports Sci. 2017 Dec;35(23):231321.

2. Van Hooren B, Bosch F. Is there really an eccentric action of the hamstrings during the swing phase of high-speed running? Part II: Implications for exercise. J Sports Sci. 2017 Dec;35(23):2322-33.

3. Van Hooren B, Bosch F. Preventing hamstring injuries-Part 2: There is possibly an isometric action of the hamstrings in high-speed running and it does matter. Sport Perf Sci Rep. 2018;1. 4. Chumanov ES, Heiderscheit BC, Thelen DG. The effect of speed and influence of individual muscles on hamstring mechanics during the swing phase of sprinting. J Biomech. 2007;40(16):3555-62.
5. Thelen DG, Chumanov ES, Best TM, Swanson SC, Heiderscheit BC. Simulation of biceps femoris musculotendon mechanics during the swing phase of sprinting. Med Sci Sports Exerc. 2005 Nov;37(11):1931-8.

6. Schache AG, Kim HJ, Morgan DL, Pandy MG. Hamstring muscle forces prior to and immediately following an acute sprinting-related muscle strain injury. Gait Posture. 2010 May;32(1):136-40.

7. Schache A. Eccentric hamstring muscle training can prevent hamstring injuries in soccer players. J Physiother. 2012;58(1):58.

8. Stanton P, Purdham C. Hamstring injuries in sprinting - the role of eccentric exercise. J Orthop Sports Phys Ther. 1989;10(9):343-9.

9. Liu Y, Sun Y, Zhu W, Yu J. Comments to "Mechanism of hamstring muscle strain injury in sprinting" by $\mathrm{Yu}$ et al. J Sport Health Sci. 2017 Jun;6(2):139-40.

10. Liu Y, Sun Y, Zhu W, Yu J. The late swing and early stance of sprinting are most hazardous for hamstring injuries. J Sport Health Sci. 2017 Jun;6(2):133-6.

11. Yu B, Liu H, Garrett WE. Mechanism of hamstring muscle strain injury in sprinting. J Sport Health Sci. 2017 Jun;6(2):130-2.

12. Yu B, Liu H, Garrett WE. Comment on "The late swing and early stance of sprinting are most hazardous for hamstring injuries" by Liu et al. J Sport Health Sci. 2017 Jun;6(2):137-8.

13. Yu B, Queen RM, Abbey AN, Liu Y, Moorman CT, Garrett WE. Hamstring muscle kinematics and activation during overground sprinting. J Biomech. 2008 Nov 14;41(15):3121-6.

14. Sides D. Kinematics and kinetics of maximal velocity sprinting and specificity of training in elite athletes: University of Salford; 2015.

15. Sun Y, Wei S, Zhong Y, Fu W, Li L, Liu Y. How joint torques affect hamstring injury risk in sprinting swing-stance transition. Med Sci Sports Exerc. 2015 Feb;47(2):373-80.

16. Shield AJ, \& Murphy S. . Is there really an eccentric action of the hamstrings in high speed running and does it matter? Sport Perf Sci Rep. 2018.

17. Macdonald B, O'Neill J, Pollock N, Van Hooren B. Single-Leg Roman Chair Hold Is More Effective Than the Nordic Hamstring Curl in Improving Hamstring Strength-Endurance in Gaelic Footballers With Previous Hamstring Injury. J Strength Cond Res. 2019 Dec;33(12):3302-8.

18. Oranchuk DJ, Storey AG, Nelson AR, Cronin JB. Scientific Basis for Eccentric Quasi-Isometric Resistance Training: A Narrative Review. J Strength Cond Res. 2019 Oct;33(10):2846-59.

19. Buckthorpe M, Wright S, Bruce-Low S, Nanni G, Sturdy T, Gross AS, et al. Recommendations for hamstring injury prevention in elite football: 
translating research into practice. Br J Sports Med. 2019 Apr;53(7):449-56.

20. Van Hooren B, Bosch F. Influence of muscle slack on high-intensity sport performance: a review. Strength and Conditioning Journal. 2016;38(5):75-87.

21. Hug F, Lacourpaille L, Maisetti O, Nordez A. Slack length of gastrocnemius medialis and Achilles tendon occurs at different ankle angles. J Biomech. 2013 Sep 27;46(14):2534-8.

22. Jönhagen $\mathrm{S}$, Ericson MO, Nemeth G, Eriksson E. Amplitude and timing of electromyographic activity during sprinting. Scandinavian journal of medicine \& science in sports. 1996;6(1):15-21.

23. Hakkinen $\mathrm{K}$, Kallinen $\mathrm{M}$, Izquierdo $\mathrm{M}$, Jokelainen K, Lassila H, Malkia E, et al. Changes in agonist-antagonist EMG, muscle CSA, and force during strength training in middle-aged and older people. J Appl Physiol (1985). 1998 Apr;84(4):1341-9. 24. Zhang L, Li H, Garrett WE, Liu H, Yu B. Hamstring muscle-tendon unit lengthening and activation in instep and cut-off kicking. J Biomech. 2020 Jan 23;99:109482.

25. Hogan N. Adaptive control of mechanical impedance by coactivation of antagonist muscles. IEEE Transactions on automatic control. 1984;29(8):681-90.

26. Bigland B, Lippold OC. The relation between force, velocity and integrated electrical activity in human muscles. J Physiol. 1954 Jan;123(1):214-24.

27. Zhou S, Lawson DL, Morrison WE, Fairweather I. Electromechanical delay in isometric muscle contractions evoked by voluntary, reflex and electrical stimulation. Eur J Appl Physiol Occup Physiol. 1995;70(2):138-45.

28. Cavanagh PR, Komi PV. Electromechanical delay in human skeletal muscle under concentric and eccentric contractions. Eur J Appl Physiol Occup Physiol. 1979 Nov;42(3):159-63.

29. Norman RW, Komi PV. Electromechanical delay in skeletal muscle under normal movement conditions. Acta Physiol Scand. 1979 Jul;106(3):2418 .

30. Muraoka T, Muramatsu T, Fukunaga T, Kanehisa $H$. Influence of tendon slack on electromechanical delay in the human medial gastrocnemius in vivo. J Appl Physiol (1985). 2004 Feb;96(2):540-4.

31. Katz B. The relation between force and speed in muscular contraction. J Physiol. 1939 Jun 14;96(1):45-64.

32. Friden J, Lieber RL. Structural and mechanical basis of exercise-induced muscle injury. Med Sci Sports Exerc. 1992 May;24(5):521-30.

33. Herzog W. The multiple roles of titin in muscle contraction and force production. Biophys Rev. 2018 Aug;10(4):1187-99.
34. Herzog W. The role of titin in eccentric muscle contraction. J Exp Biol. 2014 Aug 15;217(Pt 16):2825-33

35. Nishikawa KC, Monroy JA, Uyeno TE, Yeo SH, Pai DK, Lindstedt SL. Is titin a 'winding filament'? A new twist on muscle contraction. Proc Biol Sci. 2012 Mar 7;279(1730):981-90.

36. Leonard TR, Herzog W. Regulation of muscle force in the absence of actin-myosin-based crossbridge interaction. Am J Physiol Cell Physiol. 2010 Jul;299(1):C14-20.

37. Seliger V, Dolejs L, Karas V. A dynamometric comparison of maximum eccentric, concentric, and isometric contractions using emg and energy expenditure measurements. Eur J Appl Physiol Occup Physiol. 1980;45(2-3):235-44.

38. Asmussen E. Positive and negative muscular work. Acta Physiol Scand. 1953;28(4):364-82.

39. Tesch PA, Lindborg BP, Colliander EB. Evaluation of a dynamometer measuring torque of uniand bilateral concentric and eccentric muscle action. Clin Physiol. 1990 Jan;10(1):1-9.

40. Bigland-Ritchie B, Woods JJ. Integrated electromyogram and oxygen uptake during positive and negative work. J Physiol. 1976 Sep;260(2):267-77. 41. Dudley GA, Tesch PA, Harris RT, Golden CL, Buchanan P. Influence of eccentric actions on the metabolic cost of resistance exercise. Aviat Space Environ Med. 1991 Jul;62(7):678-82.

42. Caruso JF, Hernandez DA, Saito K, Cho M, Nelson NM. Inclusion of eccentric actions on net caloric cost resulting from isoinertial resistance exercise. J Strength Cond Res. 2003 Aug;17(3):54955.

43. Prilutsky B. Eccentric muscle action in sport and exercise. Biomechanics in sport: performance enhancement and injury prevention. 2000:56-86.

44. Herzog JA, Leonard TR, Jinha A, Herzog W. Are titin properties reflected in single myofibrils? J Biomech. 2012 Jul 26;45(11):1893-9.

45. Leonard TR, DuVall M, Herzog W. Force enhancement following stretch in a single sarcomere. Am J Physiol Cell Physiol. 2010 Dec;299(6):C1398401.

46. Opitz CA, Kulke M, Leake MC, Neagoe C, Hinssen H, Hajjar RJ, et al. Damped elastic recoil of the titin spring in myofibrils of human myocardium. Proc Natl Acad Sci U S A. 2003 Oct 28;100(22):12688-93.

47. Hill AV. The heat of shortening and the dynamic constants of muscle. Proc R Soc Lond B Biol Sci. 1938;126(843):136-95.

48. Hill AV. The series elastic component of muscle. Proc R Soc Lond B Biol Sci. 1950 Jul 24;137(887):273-80. 
49. Herzog W. The problem with skeletal muscle series elasticity. BMC Biomedical Engineering. 2019;1(1):1-14.

50. Hessel AL, Nishikawa KC. Effects of a titin mutation on negative work during stretch-shortening cycles in skeletal muscles. J Exp Biol. 2017 Nov 15;220(Pt 22):4177-85.

51. Suzuki S, Sugi H. Extensibility of the myofilaments in vertebrate skeletal muscle as revealed by stretching rigor muscle fibers. J Gen Physiol. 1983 Apr;81(4):531-46.

52. Sugi H, Akimoto T, Kobayashi T, Suzuki S, Shimada M. Possible contribution of titin filaments to the compliant series elastic component in horseshoe crab skeletal muscle fibers. Adv Exp Med Biol. 2000;481:371-80; discussion 81-2.

53. Gordon AM, Huxley AF, Julian FJ. The variation in isometric tension with sarcomere length in vertebrate muscle fibres. J Physiol. 1966 May;184(1):170-92.

54. Lieber RL, Baskin RJ. Intersarcomere dynamics of single muscle fibers during fixed-end tetani. J Gen Physiol. 1983 Sep;82(3):347-64.

55. Moo EK, Leonard TR, Herzog W. In Vivo Sarcomere Lengths Become More Non-uniform upon Activation in Intact Whole Muscle. Front Physiol. 2017;8:1015.

56. Horowits R, Podolsky RJ. The positional stability of thick filaments in activated skeletal muscle depends on sarcomere length: evidence for the role of titin filaments. J Cell Biol. 1987 Nov;105(5):2217-23. 57. Morgan DL. New insights into the behavior of muscle during active lengthening. Biophys J. 1990 Feb;57(2):209-21.

58. Burton K, Zagotta WN, Baskin RJ. Sarcomere length behaviour along single frog muscle fibres at different lengths during isometric tetani. J Muscle Res Cell Motil. 1989 Feb;10(1):67-84.

59. Edman KA, Reggiani C. Redistribution of sarcomere length during isometric contraction of frog muscle fibres and its relation to tension creep. J Physiol. 1984 Jun;351:169-98.

60. Ahn AN, Monti RJ, Biewener AA. In vivo and in vitro heterogeneity of segment length changes in the semimembranosus muscle of the toad. J Physiol. 2003 Jun 15;549(Pt 3):877-88.

61. Pappas GP, Asakawa DS, Delp SL, Zajac FE, Drace JE. Nonuniform shortening in the biceps brachii during elbow flexion. J Appl Physiol (1985). 2002 Jun;92(6):2381-9.

62. Woodley SJ, Mercer SR. Hamstring muscles: architecture and innervation. Cells Tissues Organs. 2005;179(3):125-41.

63. Timmins R, Woodley S, Shield A, Opar D. Anatomy of the Hamstrings. Prevention and Rehabilitation of Hamstring Injuries: Springer; 2020. p. 1-30.
64. Perumal R, Wexler AS, Ding J, BinderMacleod SA. Modeling the length dependence of isometric force in human quadriceps muscles. J Biomech. 2002 Jul;35(7):919-30.

65. Walshe AD, Wilson GJ, Ettema GJ. Stretchshorten cycle compared with isometric preload: contributions to enhanced muscular performance. J Appl Physiol (1985). 1998 Jan;84(1):97-106.

66. Lieberman DE, Raichlen DA, Pontzer H, Bramble DM, Cutright-Smith E. The human gluteus maximus and its role in running. J Exp Biol. 2006 Jun;209(Pt 11):2143-55.

67. Ono T, Higashihara A, Fukubayashi T. Hamstring functions during hip-extension exercise assessed with electromyography and magnetic resonance imaging. Res Sports Med. 2011 Jan;19(1):42-52.

68. Thelen DG, Chumanov ES, Hoerth DM, Best TM, Swanson SC, Li L, et al. Hamstring muscle kinematics during treadmill sprinting. Med Sci Sports Exerc. 2005 Jan;37(1):108-14.

69. Morin J. Sprint running mechanics. New technology, new concepts, new perspectives. Aspetar Sports Med J. 2013;2(3):326-32.

70. Lord JP, Aitkens SG, McCrory MA, Bernauer EM. Isometric and isokinetic measurement of hamstring and quadriceps strength. Arch Phys Med Rehabil. 1992 Apr;73(4):324-30.

71. Mohamed O, Perry J, Hislop H. Relationship between wire EMG activity, muscle length, and torque of the hamstrings. Clin Biomech (Bristol, Avon). 2002 Oct;17(8):569-79.

72. Fulton AB, Isaacs WB. Titin, a huge, elastic sarcomeric protein with a probable role in morphogenesis. Bioessays. 1991 Apr;13(4):157-61.

73. Horowits R, Kempner ES, Bisher ME, Podolsky RJ. A physiological role for titin and nebulin in skeletal muscle. Nature. 1986 Sep 1117;323(6084):160-4.

74. Labeit D, Watanabe K, Witt C, Fujita H, Wu Y, Lahmers S, et al. Calcium-dependent molecular spring elements in the giant protein titin. Proc Natl Acad Sci U S A. 2003 Nov 11;100(23):13716-21.

75. Gregersen CS, Silverton NA, Carrier DR. External work and potential for elastic storage at the limb joints of running dogs. J Exp Biol. 1998 Dec;201(Pt 23):3197-210.

76. Gillis GB, Flynn JP, McGuigan P, Biewener AA. Patterns of strain and activation in the thigh muscles of goats across gaits during level locomotion. J Exp Biol. 2005 Dec;208(Pt 24):4599-611.

77. Williams SB, Wilson AM, Daynes J, Peckham K, Payne RC. Functional anatomy and muscle moment arms of the thoracic limb of an elite sprinting athlete: the racing greyhound (Canis familiaris). J Anat. 2008 Oct;213(4):373-82. 
78. Wentink GH. The action of the hind limb musculature of the dog in walking. Acta Anat (Basel). 1976;96(1):70-80. 